\title{
DUALITY THEOREMS FOR CONVEX PROGRAMMING WITHOUT CONSTRAINT QUALIFICATION
}

\author{
P. KANNIAPPAN \\ (Received 18 January 1982; revised 9 August 1982) \\ Communicated by B. Mond
}

\begin{abstract}
Invoking a recent characterization of Optimality for a convex programming problem with finite dimensional range without any constraint qualification given by Borwein and Wolkowicz, we establish duality theorems. These duality theorems subsume numerous earlier duality results with constraint qualifications. We apply our duality theorems in the case of the objective function being the sum of a positively homogeneous, lower-semi-continuous, convex function and a subdifferentiable convex function. We also study specific problems of the above type in this setting.
\end{abstract}

1980 Mathematics subject classification (Amer. Math. Soc.): 90 C 25, 90 C 30, 90 C 48.

\section{Introduction}

We consider the following pair of problems:

Problem (P). Minimize $f(x)$ subject to

$$
g_{i}(x) \leqslant 0, \quad i=1, \ldots, m \text { and } x \in C .
$$

Problem (D). Maximize $f(x)+y^{t} g(x)$ subject to

$$
y \geqslant 0, \quad x \in C \text { and }
$$

$$
0 \in \partial f(x)+\Sigma_{m}^{1} y_{i} \partial g_{i}(x)+N(x / C) \text {. }
$$

The research was supported by the University Grants Commission of India.

(C) 1984 Australian Mathematical Society 0263-6115/84\$A2.00+0.00 
Here $f$ and $g_{i}$ are continuous convex function on a real locally convex space $X$. $C$ is a convex subset of $X$ and $\partial$ denotes subdifferential. $y=\left(y_{1}, \ldots, y_{m}\right)$ is a vector in $R^{m}$ and $t$ denotes transpose. $N(x / C)$ denotes the normal cone to $C$ at $x$ defined by

$$
N(x / C)=\left\{y \in X^{*}:\langle y, z-x\rangle \leqslant 0, \text { for every } z \in C\right\}
$$

where $X^{*}$ is the topological dual space of $X$.

Recently, Schechter [19] established a duality theorem between the problems (P) and (D) assuming Slater's Constraint qualification, namely, there exists an $x^{\prime} \in C$ such that $g_{i}\left(x^{\prime}\right)<0$, for $i=1, \ldots, m$. His duality theorem [19, Theorem $2.2]$ is the following.

THEOREM 1.1. Suppose that $\bar{x}$ is optimal for problem (P). Then there exists $\bar{y}$ such that $(\bar{x}, \bar{y})$ is optimal for problem (D) and the two problems have the same extremal values.

If $f$ and $g_{i}, i=1, \ldots, m$, are differentiable, $X=R^{n}$ and $C=X$, then (1.1) becomes

$$
\nabla f(x)+\sum_{1}^{m} y_{i} \nabla g_{i}(x)=0, \quad y \geqslant 0
$$

where $\nabla$ denotes gradient vector and Theorem 1.1 becomes the duality theorem of Wolfe [21], where he has assumed the constraint qualification of Kuhn and Tucker [10, page 483].

Recently, Ben-Israel, Ben-Tal and Zlobec $[2,3]$ established necessary and sufficient conditions for a vector $x_{0}$ to be optimal for problem (P) without the need for a constraint qualification when $X$ is a finite dimensional space. Their results are called 'BBZ' conditions. Using 'BBZ' conditions, Mond and Zlobec established duality theorems in [13]. More recently, the above characterization for optimality was established by Borwein and Wolkowicz [4] in a general locally convex space without assuming any constraint qualification. In this paper we utilize the results of Borwein and Wolkowicz [4] to establish duality results without assuming any constraint qualification. Duality results in [18], including those of Wolfe [21] and Schechter [19], are special cases of our duality results. In Section 3, we also give a converse duality theorem. In Section 4, we apply our duality results to the case where the objective function is the sum of a positively homogeneous, lower-semi-continuous, convex function and a subdifferentiable convex function. Finally, we reformulate many other duality results in the literature so as to eliminate the need for any constraint qualification. 


\section{Preliminaries}

In this paper we consider the convex programming problem:

Problem $\left(\mathrm{P}_{1}\right)$. Minimize $p(x)$ subject to $g(x) \in-S$ and $x \in C$

where $p: X \rightarrow R \cup\{+\infty\}, g: X \rightarrow Y \cup\{+\infty\} ; X$ and $Y$ are real locally convex spaces; $Y$ is finite dimensional with an abstract maximal element $+\infty$ (see [14]); $C \subset X$ and $S \subset Y$ are convex and $S$ is also a cone; $p$ is a convex functional and $g$ is $S$-convex (on $C$ ), that is,

$$
\operatorname{tg}\left(x_{1}\right)+(1-t) g\left(x_{2}\right)-g\left(t x_{1}+(1-t) x_{2}\right) \in S,
$$

for any $x_{1}, x_{2} \in C$ and $0 \leqslant t \leqslant 1$.

The convex cone $S$ induces a partial ordering ' $\geqslant$ ' on $Y$ given on

$$
x_{1} \geqslant x_{2} \Leftrightarrow x_{1}-x_{2} \in S \text {. }
$$

We make the additional routine assumption that the feasible set

$$
A=g^{-1}(-S) \cap C
$$

is a non-empty subset of $\operatorname{dom} p$, where $\operatorname{dom} p=\{x \in X: p(X)<\infty\}$. Let $X^{*}$ and $Y^{*}$ denote the continuous dual spaces of $X$ and $Y$, respectively. We suppose throughout that $X^{*}$ is endowed with the weak star topology $\sigma\left(X^{*}, X\right)$ (see [15] for details). We write $\left\langle x^{*}, x\right\rangle$ for the value of the continuous linear functional $x^{*} \in X^{*}$ at the point $x \in X$.

Given any set $K$ in $X$, the dual cone of $K$ is the set in $X^{*}$

$$
K^{+}=\left\{x^{*} \in X^{*}:\left\langle x^{*}, x\right\rangle \geqslant 0 \text {, for every } x \in K\right\} .
$$

We denote the annihilator of a set $K$ in $X$ by $K^{\perp}=K^{+} \cap\left(-K^{+}\right)$and the convex cone generated by $K$ by cone $K$.

A continuous linear operator $T: X \rightarrow Y$ is a subgradient for $g$ at $a$ if

$$
T(x-a) \leqslant g(x)-g(a) \text { for every } x \in X .
$$

The set of all such subgradients is denoted by $\partial g(a)$.

The $S$-convex operator $g: X \rightarrow Y$ is said to be regularly-sub-differentiable at $a$ if

$$
\partial\left(x^{*} \circ g\right)(a)=x^{*} \circ \partial g(a)
$$

for every $x^{*} \in S^{*}$. It is easy to see that, if $g$ is continuous and differentiable at $a$, that is $\partial g(a)$ contains a unique element (for instance if $g$ is Fréchet differentiable), then $g$ is regularly subdifferentiable at $a$. For more details about regular subdifferentiability, the reader can refer to $[1,22]$. 
$K$ is a face of a convex cone $S$ if $K$ is a convex cone, and $s_{1}, s_{2} \in S$, $s_{1}+s_{2} \in K \Rightarrow s_{1}, s_{2} \in K$. We denote by $S^{f}$, the (unique) smallest face of $S$ which contains $-g(A)$.

Let $a \in A$. We define

$$
D_{g}^{<}(a)=\left\{d: \text { there exists } \alpha>0 \text { with } g(a+t d) \in S^{f}-S \text { if } 0 \leqslant t \leqslant \alpha\right\} .
$$

If $Y=R^{m}$ and $S=R_{+}^{m}$, then (2.1) corresponds to the intersection of the cones of non-increase of the equality constraints, that is, the constraints which are identically zero on the feasible set $[2,3]$.

\section{Duality and converse duality theorems}

The following theorem can be proved using the definition of regular subdifferentiability and Corollaries 4.2 and 4.3 of Borwein and Wolkowicz [4] (see also Zowe [22]).

THEOREM 3.1. Suppose that $S^{+}+\left(S^{f}\right)^{\perp}=\left(S^{f}\right)^{+}$holds. Suppose also that $f$ is a convex functional and $g$ is $S$-convex on $X$ and that they are continuous at a. Further, we assume that $g$ is regularly subdifferentiable at a. Then $a \in A$ is optimal for $\left(\mathrm{P}_{1}\right)$ if and only if there exists $s^{+} \in S^{+}$such that

$$
0 \in \partial p(a)+s^{+} \partial g(a)-\left(D_{g}^{\leftarrow}(a) \cap \operatorname{Cone}(C-a)\right)^{+} \text {and } s^{+} g(a)=0 .
$$

Based on the above characterization of optimality without any constraint qualification, we can formulate the following dual problem $\left(D_{1}\right)$ to the primal problem $\left(\mathrm{P}_{1}\right)$.

Problem $\left(\mathrm{D}_{1}\right)$. Maximize $p(x)+s^{+} g(x)$ subject to

$s^{+} \in S^{+}, x \in A^{f}$ and

$0 \in \partial p(x)+s^{+} \partial g(x)-\left(D_{g}^{<}(x) \cap \operatorname{Cone}(C-x)\right)^{+}$

where $A^{f}=g^{-1}\left(S^{f}-S\right) \cap C$.

Note. $A \subset A^{f}$. Further $D_{g}^{<}(x) \cap \operatorname{Cone}(C-x)$ is the smallest cone containing $A^{f}$ (see [4] for details).

Now, we establish the duality theorem between the problems $\left(P_{1}\right)$ and $\left(D_{1}\right)$ without any constraint qualification. 
THEOREM 3.2 (duality). Let us assume the hypothesis of Theorem 3.1. If $a_{0} \in A$ is optimal for problem $\left(\mathrm{P}_{1}\right)$, then there exists $s_{0}^{+} \in S^{+}$such that $\left(a_{0}, s_{0}^{+}\right)$is optimal for problem $\left(\mathrm{D}_{1}\right)$ and furthermore the two problems have the same extremal values.

Proof. Since $a_{0} \in A$ is optimal for $\left(\mathrm{P}_{1}\right)$, Theorem 3.1 guarantees the existence of feasible solutions to problem $\left(D_{1}\right)$.

Let $\left(a, s^{+}\right)$be a feasible solution for problem $\left(\mathrm{D}_{1}\right)$. Then $a \in A^{f}, s^{+} \in S^{+}$and

$$
0 \in \partial p(a)+s^{+} \partial g(a)-\left(D_{g}^{\leftarrow}(a) \cap \operatorname{Cone}(C-a)\right)^{+} .
$$

This implies that there exist $v^{*} \in \partial p(a)$ and $T \in \partial g(a)$ and $w^{*} \in\left(D^{\leqslant}(a) \cap\right.$ Cone $(C-a))^{+}$such that

$$
0=v^{*}+s^{+} T-w^{*}
$$

Now, consider

$$
\begin{aligned}
p\left(a_{0}\right)-\left[p(a)+s^{+} g(a)\right] & =\left[p\left(a_{0}\right)-p(a)\right]-s^{+} g(a) \\
& \geqslant\left\langle v^{*}, a_{0}-a\right\rangle-s^{+} g(a), \text { since } v^{*} \in \partial p(a) \\
& =\left\langle-s^{+} T+w^{*}, a_{0}-a\right\rangle-s^{+} g(a), \quad \text { from }(3.1) \\
& =\left\langle-s^{+} T, a_{0}-a\right\rangle+\left\langle w^{*}, a_{0}-a\right\rangle-s^{+} g(a) \\
& \geqslant\left\langle s^{+}, g(a)-g\left(a_{0}\right)\right\rangle+\left\langle w^{*}, a_{0}-a\right\rangle-s^{+} g(a), \\
& =-s^{+} g\left(a_{0}\right)+\left\langle w^{*}, a_{0}-a\right\rangle \\
& \geqslant 0,
\end{aligned}
$$

since $s^{+} \in S^{+}, g\left(a_{0}\right) \in-S$ and $w^{*} \in\left(D_{g}^{<}(a) \cap \text { Cone }(C-a)\right)^{+}=\partial I_{A}(A)$ (see [16], Corollary 23.5.4), where $I_{A^{\prime}}$ is the indicator function of $A^{f}$; that is, $I_{A^{\prime}}(x)$ is 0 on $A^{f}$ and $+\infty$ elsewhere. Thus,

$$
p\left(a_{0}\right) \geqslant p(a)+s^{+} g(a)
$$

for every feasible solution $\left(a, s^{+}\right)$of $\left(\mathrm{D}_{1}\right)$.

Since $a_{0}$ is an optimal solution of $\left(\mathrm{P}_{1}\right)$, we have from Theorem 3.1 , that there exists $s_{0}^{+} \in S^{+}$such that $s_{0}^{+} g\left(a_{0}\right)=0$ and

$$
0 \in \partial p\left(a_{0}\right)+s_{0}^{+} \partial g\left(a_{0}\right)-\left(D_{g}^{\leqslant}\left(a_{0}\right) \cap \operatorname{Cone}\left(C-a_{0}\right)\right)^{+} .
$$

In other words, $\left(a_{0}, s_{0}^{+}\right)$is a feasible solution for $\left(\mathrm{D}_{1}\right)$ and

$$
p\left(a_{0}\right)=p\left(a_{0}\right)+s_{0}^{+} g\left(a_{0}\right) \text {. }
$$

Hence, from (3.2) and (3.3), $\left(a_{0}, s_{0}^{+}\right)$is an optimal solution of $\left(D_{1}\right)$ and that the extremal values of the two problems $\left(P_{1}\right)$ and $\left(D_{1}\right)$ are equal. 
REMARKS 3.3. Lemma 3.1(a) in [4] shows that when the convex cone $S$ has non-empty interior, then $S^{f}=S$ exactly when Slater's constraint qualification holds for $\left(\mathrm{P}_{1}\right)$, that is, there exists $x \in C$ with $g(x) \in$-int $S$, where int denotes interior. Thus, we can draw the following conclusions.

(a) If Slater's constraint qualification is satisfied for $\left(\mathrm{P}_{1}\right)$, then $S^{f}=S$ and in this case $D_{g}^{<}(a)=X$ and $D_{g}^{<}(a) \cap \operatorname{Cone}(C-a)=\operatorname{Cone}(C-a)$. Hence $\left(D_{g}^{\leftarrow}(a) \cap \operatorname{Cone}(C-a)\right)^{+}=(\operatorname{Cone}(C-a))^{+}=N(a / C)$ (see Holmes [6], page 24).

When $Y=R^{m}, S=R_{+}^{m}$, problem $\left(D_{1}\right)$ now reduces to problem (D) and Theorem 3.2 yields Schechter's duality theorem [19], that is, Theorem 1.1 in the introduction.

(b) In addition to the assumptions in (a), if we further assume that $X=R^{n}$, $C=X$, then $\left.D_{g}^{<}(a) \cap \operatorname{Cone}(C-a)\right)^{+}=\{0\}$. Problem $\left(D_{1}\right)$ becomes

Maximize $p(x)+s^{t} g(x)$ subject to

$$
s \geqslant 0 \text { and } 0 \in \partial p(x)+s^{t} \partial g(x)
$$

and Theorem 3.2 becomes Schechter's duality theorem 1 in [18].

(c) In addition to the conditions in (b) if $p$ and $g$ are differentiable, then problem $\left(D_{1}\right)$ becomes

$$
\begin{aligned}
& \text { Maximize } p(x)+s^{t} g(x) \text { subject to } \\
& \qquad s \geqslant 0, \quad \nabla p(x)+s^{+} \nabla g(x)=0
\end{aligned}
$$

and Wolfe's duality theorem 2 in [21] in recovered from Theorem 3.2.

Assuming Slater's constraint qualification a duality theorem in the spirit of Schechter $[18,19]$ has been proved in a locally convex space with operatorial constraints in $[8,9]$.

We shall now prove a converse duality theorem between the problems $\left(P_{1}\right)$ and $\left(D_{1}\right)$ without any constraint qualification.

THEOREM 3.3 (converse duality). Let us assume the hypothesis of Theorem 3.1 and assume that the problem $\left(\mathrm{P}_{1}\right)$ has a solution $\bar{a}$. If $\left(a_{0}, s_{0}^{+}\right)$is an optimal solution of the problem $\left(\mathrm{D}_{1}\right)$, and if $p$ is strictly convex at $a_{0}$, then $a_{0}=\bar{a}$. Hence $a_{0}$ solves the problem $\left(\mathrm{P}_{1}\right)$. Furthermore, the extremal values of the two problems are the same. 
Proof. Supposing $a_{0} \neq \bar{a}$. Since $\bar{a}$ is a solution of $\left(\mathrm{P}_{1}\right)$, it follows from Theorem 3.1 that there exists $\bar{s}^{+} \in S^{+}$such that

$$
0 \in \partial p(\bar{a})+\bar{s}^{+} \partial g(\bar{a})-\left(D_{g}^{<}(\bar{a}) \cap \operatorname{Cone}(C-\bar{a})\right)^{+}
$$

and

$$
\bar{s}^{+} g(\bar{a})=0 .
$$

Let $L\left(a, s^{+}\right)=p(a)+s^{+} g(a)$ be the Lagrangian of $\left(\mathrm{P}_{1}\right)$. Then

$$
L\left(\bar{a}, \bar{s}^{+}\right)=L\left(a_{0}, s_{0}^{+}\right)=\max _{\left(a, s^{+}\right) \in N} L\left(a, s^{+}\right)
$$

where $N=\left\{\left(a, s^{+}\right): a \in A^{f}, s^{+} \in S^{+}\right.$and $0 \in \partial p(a)+s^{+} \partial g(a)-\left(D_{g}^{\leftarrow}(a) \cap\right.$ Cone $\left.(C-a))^{+}\right\}$. Note that $\left(\bar{a}, \bar{s}^{+}\right) \in N$.

Since $\left(a_{0}, s_{0}^{+}\right) \in N$, we have $0 \in \partial p\left(a_{0}\right)+s_{0}^{+} \partial g\left(a_{0}\right)-\left(D_{g}^{<}\left(a_{0}\right) \cap \operatorname{Cone}(C\right.$ $\left.\left.-a_{0}\right)\right)^{+}$. Hence there exist $u^{*} \in \partial p\left(a_{0}\right), T \in \partial g\left(a_{0}\right)$ and $w^{*} \in\left(D_{g}^{<}\left(a_{0}\right) \cap\right.$ Cone $\left.\left(C-a_{0}\right)\right)^{+}$such that

$$
0=u^{*}+s_{0}^{+} T-w^{*} .
$$

Now, consider

$$
\begin{aligned}
L\left(\bar{a}, s_{0}^{+}\right)-L\left(a_{0}, s_{0}^{+}\right) & =p(\bar{a})+s_{0}^{+} g(\bar{a})-p\left(a_{0}\right)-s_{0}^{+} g\left(a_{0}\right) \\
& =\left[p(\bar{a})-p\left(a_{0}\right)\right]+s_{0}^{+}\left[g(\bar{a})-g\left(a_{0}\right)\right] \\
& \rangle\left\langle u^{*}, \bar{a}-a_{0}\right\rangle+\left\langle s_{0}^{+}, g(\bar{a})-g\left(a_{0}\right)\right\rangle, \\
& \quad \text { since } p \text { is strictly convex at } a_{0} \\
& \geqslant\left\langle u^{*}, \bar{a}-a_{0}\right\rangle+\left\langle s_{0}^{+}, T(\bar{a})-T\left(a_{0}\right)\right\rangle, \\
& \text { since } T \in \partial g\left(a_{0}\right) \\
& =\left\langle u^{*}, \bar{a}-a_{0}\right\rangle+\left\langle s_{0}^{+} T, \bar{a}-a_{0}\right\rangle \\
& =\left\langle w^{*}, \bar{a}-a_{0}\right\rangle \geqslant 0, \quad \text { by (3.4). }
\end{aligned}
$$

since $w^{*} \in\left(D_{g}^{<}\left(a_{0}\right) \cap \operatorname{Cone}\left(C-a_{0}\right)\right)^{+}$(see the proof of Theorem 3.2). It follows that, $L\left(\bar{a}, s_{0}^{+}\right)>L\left(a_{0}, s_{0}^{+}\right)=L\left(\bar{a}, \bar{s}^{+}\right)$. That is,

$$
p(\bar{a})+s_{0}^{+} g(\bar{a})>p(\bar{a})+\bar{s}^{+} g(\bar{a}) .
$$

Hence, (3.6) becomes by (3.4), $s_{0}^{+} g(\bar{a})>0$, which is a contradiction to the fact that $s_{0}^{+} \in S^{+}, g(\bar{a}) \in-S$. Hence $\bar{a}=a_{0}$ and $a_{0}$ solves problem $\left(\mathrm{P}_{1}\right)$.

Furthermore, we have

$$
\begin{aligned}
p\left(a_{0}\right) & =p(\bar{a})=p(\bar{a})+\bar{s}^{+} g(\bar{a})=L\left(\bar{a}, \bar{s}^{+}\right)=L\left(a_{0}, s_{0}^{+}\right) \\
& =p\left(a_{0}\right)+s_{0}^{+} g\left(a_{0}\right) .
\end{aligned}
$$

Hence the extremal values of the two problems are equal. 
EXAMPLE. The following is an example of a minimization problem where the Slater's constraint qualification is not satisfied. We will show that Wolfe's duality theorem is not applicable to this example. However, the duality theorem of this section can be applied.

Problem $\left(\mathbf{P}^{\prime}\right)$. Minimize $p(x)=-x$ subject to

$$
g(x) \leqslant 0
$$

where $g(x)=x^{2}$ if $x \geqslant 0$ and $g(x)=0$ otherwise.

The feasible set $F$ for this problem $\left(\mathrm{P}^{\prime}\right)$ is

$$
F=\{x \in R: g(x) \leqslant 0\}=(-\infty, 0] .
$$

Clearly $x=0$ is the optimal solution of problem $\left(P^{\prime}\right)$. The functions $p$ and $g$ are differentiable everywhere. The derivative of $g$ at $x$ is denoted by $g^{\prime}(x)$, where

$$
g^{\prime}(x)= \begin{cases}2 x & \text { if } x \geqslant 0 \\ -1 & \text { if } x<0\end{cases}
$$

Corresponding to the primal problem $\left(\mathrm{P}^{\prime}\right)$, the Wolfe's [21] dual problem $\left(\mathrm{D}^{\prime}\right)$ is

Problem $\left(\mathrm{D}^{\prime}\right)$. Maximize $-x+\lambda x^{2}$ subject to

$$
x \in F, \lambda \geqslant 0, \quad-1+\lambda g^{\prime}(x)=0 .
$$

For any $x \in F, \lambda \geqslant 0$, the condition (3.7) is not satisfied, which implies feasible solutions for problem $\left(\mathrm{D}^{\prime}\right)$ do not exist and hence there is no optimal solution for problem $\left(\mathrm{D}^{\prime}\right)$. Wolfe's duality theorem does not hold in the case.

It is clear that $S^{f}=\{0\}$ and the condition that $S^{+}+\left(S^{f}\right)^{\perp}=\left(S^{f}\right)^{+}$is also satisfied.

By definition

$$
\begin{aligned}
D_{g}^{<}(x) & =\left\{d: \exists \alpha>0 \text { with } g(x+t d) \in S^{f}-S, 0 \leqslant t \leqslant \alpha\right\} \\
& =\{d: \exists \alpha>0 \text { with } g(x+t d) \in(-\infty, 0], 0 \leqslant t \leqslant \alpha\} \\
& =R \text { if } x<0 \\
& =(-\infty, 0] \quad \text { if } x=0 \\
& =\text { empty set if } x>0 .
\end{aligned}
$$

As per the dual problem $\left(D_{1}\right)$ in this section, the dual problem $\left(D^{\prime \prime}\right)$ in this case is:

Problem $\left(\mathrm{D}^{\prime \prime}\right) . \quad$ Maximize $-x+\lambda x^{2}$ subject to

$$
\begin{aligned}
& x \in F, \lambda \in S^{+}=[0, \infty) \text { and } \\
& -1+g^{\prime}(x) \in\left(D_{g}^{<}(x)\right)^{+} .
\end{aligned}
$$


It is clear that the set of feasible solutions for problem $\left(\mathrm{D}^{\prime \prime}\right)$ is $\{(x, \lambda): x=0$ and $\lambda \geqslant 0\}$ and any feasible solution is an optimal solution of problem $\left(\mathrm{D}^{\prime \prime}\right)$. Further the optimal value zero is same for the two problems $\left(\mathrm{P}^{\prime}\right)$ and $\left(\mathrm{D}^{\prime \prime}\right)$.

\section{Applications}

We shall now apply the theorems in Section 3 to the case where the objective function is the sum of a positively homogeneous, lower-semi-continuous, convex function and a continuous convex function. Specific problems of this type are studied in detail in $[11,12]$ assuming a constraint qualification such as Slater's type. In the next section, we shall examine the forms which the dual problem takes in these special cases without a constraint qualification.

We shall need the following definition and propositions whose proofs can be found in $[7,8]$.

Definition 4.1. Let $V$ be a non-empty subset of a locally convex space $X^{*}$. Then the support function of $V$, denoted by $s(\cdot / V)$ is defined by

$$
s(u / V)=\sup \left\{\left\langle u^{*}, u\right\rangle: u^{*} \in V\right\} \text {. }
$$

Note. Let $p$ be a positively homogeneous, lower-semi-continuous, convex function, defined on a locally convex space $X$. Then

$$
\partial p(0)=\left\{u^{*} \in X^{*}: p(u) \geqslant\left\langle u^{*}, u\right\rangle \text { for all } u \in X\right\},
$$

since $p(0)=0$.

Proposition 4.2. Let $p$ be a positively homogeneous, lower-semi-continuous, convex function defined on a locally convex space $X$. Then $p$ is the support function $\partial p(0)$.

REMARK 4.3. If $p$ is a positively homogeneous lower-semi-continuous, convex function, then $\partial p(0)$ is a non-empty, convex, subset of $X^{*}$ (see [7], page 3). In fact, there is a one to one correspondence between convex subsets of $X^{*}$ and positively homogeneous, lower-semi-continuous, convex functions on $X$.

Proposition 4.4 Let $p$ be a positively homogeneous, lower-semi-continuous convex function defined on a locally convex space $X$ and let $u \neq 0$. Then

$$
\partial p(u)=\left\{u^{*} \in \partial p(0): p(u)=\left\langle u^{*}, u\right\rangle\right\}
$$


Let the objective function $p: X \rightarrow R$ be of the form $p=p_{1}+p_{2}$, where $p_{1}$ is a continuous convex function and $p_{2}$ is a positively homogeneous, lower-semi-continuous, convex function.

The primal problem $\left(\mathrm{P}_{2}\right)$ is

Problem $\left(\mathrm{P}_{2}\right)$. Minimize $p_{1}(x)+p_{2}(x)$ subject to

$$
g(x) \in-S \text { and } x \in C \text {. }
$$

Let $\left(D_{2}\right)$ denote the following dual problem.

Problem $\left(\mathrm{D}_{2}\right)$. Maximize $p_{1}(x)+\left\langle x^{*}, x\right\rangle+s^{+} g(x)$ subject to $s^{+} \in S, x \in A^{f}, x^{*} \in \partial p_{2}(0)$ and $0 \in \partial p_{1}(x)+x^{*}+s^{*} \partial g(x)-\left(D_{g}^{<}(x) \cap \operatorname{Cone}(C-x)\right)^{+}$.

We shall now prove a duality theorem between the problems $\left(P_{2}\right)$ and $\left(D_{2}\right)$.

THEOREM 4.5. Let us assume the hypothesis of Theorem 3.1 If $a_{0} \in A$ is optimal for $\left(\mathrm{P}_{2}\right)$, then there exist $x_{0}^{*}$ and $s_{0}^{+}$such that $\left(a_{0}, x_{0}^{*}, s_{0}^{+}\right)$is optimal for $\left(\mathrm{D}_{2}\right)$.

PROof. Since $a_{0} \in A$ is optimal for $\left(\mathrm{P}_{2}\right)$, by Theorem 3.1, there exists $s^{+} \in S^{+}$ such that $s^{+} g\left(a_{0}\right)=0$ and

$$
0 \in \partial\left(p_{1}+p_{2}\right)\left(a_{0}\right)+s^{+} \partial g\left(a_{0}\right)-\left(D_{g}^{\varangle}\left(a_{0}\right) \cap \operatorname{Cone}\left(C-a_{0}\right)\right)^{+} .
$$

But $\partial\left(p_{1}+p_{2}\right)\left(a_{0}\right)=\partial p_{1}\left(a_{0}\right)+\partial p_{2}\left(a_{0}\right)$ by the Moreau-Rockafellar Theorem [16]. Also,

$$
\partial p_{2}\left(a_{0}\right)=\left\{x^{*} \in \partial p_{2}(0): p_{2}\left(a_{0}\right)=\left\langle x^{*}, a_{0}\right\rangle\right\} .
$$

Therefore, by Proposition 4.4,

$$
\begin{aligned}
0 \in & \partial p_{1}\left(a_{0}\right)+\left\{x^{*} \in \partial p_{2}(0): p_{2}\left(a_{0}\right)=\left\langle x^{*}, a_{0}\right\rangle\right\} \\
& +s^{+} \partial g\left(a_{0}\right)-\left(D_{g}^{<}\left(a_{0}\right) \cap \operatorname{Cone}\left(C-a_{0}\right)\right)^{+} .
\end{aligned}
$$

Hence, there is $x^{*} \in \partial p_{2}(0)$ satisfying $p_{2}\left(a_{0}\right)=\left\langle x^{*}, a_{0}\right\rangle$ such that

$$
0 \in \partial p_{1}\left(a_{0}\right)+x^{*}+s^{+} \partial g\left(a_{0}\right)-\left(D_{g}^{\leftarrow}\left(a_{0}\right) \cap \operatorname{Cone}\left(C-a_{0}\right)\right)^{+} .
$$

Thus, feasible solutions to problem $\left(\mathrm{D}_{2}\right)$ exist.

As in the proof of Theorem 3.2, if $\left(a, x^{*}, s^{+}\right)$is any feasible solution for $\left(\mathrm{D}_{2}\right)$, then we can prove the following inequality by invoking the definition of support 
function, the convexity of the functions $p_{1}$ and $g$ and using the Propositions 4.2 and 4.4 ,

$$
p_{1}\left(a_{0}\right)+p_{2}\left(a_{0}\right) \geqslant p_{1}(a)+\left\langle x^{*}, a\right\rangle+s^{+} g(a)
$$

for every feasible solution $\left(a, x^{*}, s^{+}\right)$of $\left(\mathrm{D}_{2}\right)$. Now, since $a_{0}$ is optimal for $\left(\mathrm{P}_{2}\right)$, there exists $s_{0}^{+} \in S^{+}, x_{0}^{*} \in \partial p_{2}(0)$ satisfying $p_{2}\left(a_{0}\right)=\left\langle x_{0}^{*}, a_{0}\right\rangle$ such that

$$
0 \in \partial p_{1}\left(a_{0}\right)+x_{0}^{*}+s_{0}^{+} \partial g\left(a_{0}\right)-\left(D_{g}^{<}\left(a_{0}\right) \cap \operatorname{Cone}\left(C-a_{0}\right)\right)^{+}
$$

and such that $s_{0}^{+} g\left(a_{0}\right)=0$. Therefore

$$
p_{1}\left(a_{0}\right)+p_{2}\left(a_{0}\right)=p_{1}\left(a_{0}\right)+p_{2}\left(a_{0}\right)+s_{0}^{+} g\left(a_{0}\right) \text {. }
$$

Hence, by (3.6), $p_{1}\left(a_{0}\right)+\left\langle x_{0}^{*}, a_{0}\right\rangle+s_{0}^{+} g\left(a_{0}\right)=p_{1}\left(a_{0}\right)+p_{2}\left(a_{0}\right)+s_{0}^{+} g\left(a_{0}\right) \geqslant$ $p_{1}(a)+\left\langle x^{*}, a\right\rangle+s^{+} g(a)$ for every feasible solution $\left(a, x^{*}, s^{+}\right)$of $\left(\mathrm{D}_{2}\right)$, which implies that $\left(a_{0}, x_{0}^{*}, s_{0}^{+}\right)$is optimal for $\left(\mathrm{D}_{2}\right)$. Further, it is clear that

$$
p_{1}\left(a_{0}\right)+p_{2}\left(a_{0}\right)=p_{1}\left(a_{0}\right)+\left\langle x_{0}^{*}, a_{0}\right\rangle+s_{0}^{+} g\left(a_{0}\right),
$$

since $p_{2}\left(a_{0}\right)=\left\langle x_{0}^{*}, a_{0}\right\rangle$ and $s_{0}^{+} g\left(a_{0}\right)=0$. Hence, the extremal values of the two problems are the same.

\section{Special cases}

We shall now apply the theory developed in Section 4 to special cases of the problems that have been found in the literature.

(1) First we consider a programming problem whose objective function contains a norm. Such types of problems are studied by Watson in [20].

Let $X$ be a real normed linear space. Consider the problem:

Problem $\left(\mathrm{P}_{2}^{\prime}\right)$. Maximize $k(x)+\|x\|$ subject to

$$
g_{i}(x) \leqslant 0, \quad i, \ldots, m, x \in C .
$$

Here $k: X \rightarrow R$ is a continuous convex functional, $g_{i}: X \rightarrow R, i=1, \ldots, m$, are subdifferentiable convex functionals and $C$ is a convex subset of $X$. Let $g$ : $X \rightarrow R^{m}$ be defined by $g(x)=\left(g_{1}(x), \ldots, g_{m}(x)\right)$.

Now, let us formulate the dual problem:

Problem $\left(\mathrm{D}_{2}^{\prime}\right) . \quad$ Maximize $k(x)+\|x\|+y^{\prime} g(x)$ subject to $y \in R_{+}^{m}, x \in A^{f}$,$$
0 \in \partial k(x)+\partial\|x\|+\Sigma_{1}^{m} y_{i} \partial g_{i}(x)-\left(D_{g}^{<}(x) \cap\right.
$$$$
\operatorname{Cone}(C-x))^{+} \text {. }
$$ 
It is a well known fact that the norm as a function from $X$ into $R$ is a continuous convex functional which is positively homogeneous. Therefore, the norm function can be expressed as a support function of the weak star closed convex set $\partial\|0\|$, by Proposition 4.2. Furthermore, it is proved in ([7], page 46), that

$$
\partial\|x\|=\left\{\begin{array}{l}
\left\{x^{*} \in X^{*}:\left\|x^{*}\right\|=1,\left\langle x^{*}, x\right\rangle=\|x\|\right\}, \quad \text { if } x \neq 0, \\
\left\{x^{*} \in X^{*}:\left\|x^{*}\right\| \leqslant 1\right\}=B^{\prime}(0,1), \quad \text { if } x=0,
\end{array}\right.
$$

where $X^{*}$ is the topological dual of $X$.

Thus, the subdifferential of the norm, in a normed linear space, at the origin coincides with the closed unit ball of the dual space. Hence, by Proposition 4.2, the norm is the support function of the unit ball of the dual space $X^{*}$.

Hence, by the foregoing argument, the dual problem $\left(D_{2}^{\prime}\right)$ becomes

Problem $\left(\mathrm{D}_{2}^{\prime}\right)$. Maximize $k(x)+\left\langle x^{*}, x\right\rangle+y^{t} g(x)$ subject to

$$
\begin{aligned}
& y \in R_{+}^{m}, x \in A^{f}, x^{*} \in B^{\prime}(0,1) \text { and } \\
& 0 \in \partial k(x)+x^{*}+\sum_{1}^{m} y_{i} \partial g_{i}(x)-\left(D_{g}^{<}(x) \cap\right. \\
& \text { Cone }(C-x))^{+} .
\end{aligned}
$$

If we assume Slater's constraint qualification, then $\left(D_{g}^{<}(x) \cap \operatorname{Cone}(C-x)\right)^{+}$ $=N(x \mid C)$ and $A^{f}=\{0\}$ (see Holmes [6]).

REMARK 5.1. If $k$ is a zero functional, then problem $\left(\mathrm{P}_{2}^{\prime}\right)$ becomes a problem in approximation theory, that is, finding a vector of minimum norm from the convex set $K=\left\{x \in X: g_{i}(x) \leqslant 0, i=1, \ldots, m\right\} \cap C$.

(2) Let us define the function $h: R^{n} \rightarrow R$ by $h(x)=\left(x^{t} B x\right)^{1 / 2}$, where $B$ is a (symmetric) positive semi-definite $n \times n$ matrix. Then the following lemma is proved in [5].

Lemma 5.2. The function $h$ is convex, and $z \in \partial h(a)$ if and only if $z=w^{t} B$, $w^{t} B w \leqslant 1$, and $h(a)=w^{t} B a$.

Now, consider the following dual pair.

Problem $\left(\mathrm{P}_{3}^{\prime}\right)$. Minimize $k(x)+\left(x^{t} B x\right)^{1 / 2}$ subject to

$$
g_{i}(x) \leqslant 0, i=1, \ldots, m, x \in C \text {. }
$$


Problem ( $\left.\mathrm{D}_{3}^{\prime}\right)$. Minimize $k(x)+x^{\prime} B z+y^{t} g(x)$ subject to

$$
\begin{aligned}
& y \in R_{+}^{m}, x \in A^{f}, B z \in \partial h(0) \text { and } \\
& 0 \in \partial k(x)+B z+\sum_{1}^{m} y_{i} \partial g_{i}(x)-\left(D_{g}^{<}(x) \cap\right. \\
& \text { Cone }(C-x))^{+} .
\end{aligned}
$$

Here $k$ and the $g_{i}$ are convex functions on $R^{n}$. Hence, they are continuous and subdifferentiable everywhere. It is clear that $\left(x^{t} B x\right)^{1 / 2}=s(x / \partial h(0))$, where $\partial h(0)$ $=\left\{B y: y^{t} B y \leqslant 1\right\}$.

If we assume Slater's constraint qualification and $C=X$, then $\left(\mathrm{P}_{3}^{\prime}\right)$ and $\left(\mathrm{D}_{3}^{\prime}\right)$ are the dual pair studied in the differentiable case in $[11,18,19]$.

REMARK 5.3. If $A=\{0\}$, then the pair $\left(\mathrm{P}_{3}^{\prime}\right)$ and $\left(\mathrm{D}_{3}^{\prime}\right)$ becomes the duality pair in Remark 3.3(b).

\section{Acknowledgement}

The author wishes to thank Dr. Sundaram M. A. Sastry for his helpful suggestions and the referees for their many helpful comments.

\section{References}

[1] V. Barbu and Th. Precupanu, Convexity and optimization in Banach space (Sijthoff and Noordhoff International Publishers, 1978).

[2] A. Ben-Israel, A. Ben-Tal and S. Zlobec, 'Optimality conditions in convex programming', in Survey of mathematical programming, Proceedings of the IX International Symposium of Mathematical Programming, edited by A. Prekopa (Hungarian Academy of Sciences, Budapest, Hungary and North-Holland, Amsterdam, 1978).

[3] A. Ben-Tal, A. Ben-Israel and S. Zlobec, 'Characterization of optimality in convex programming without a constraint qualification', J. Optimization Theory Appl. 20 (1976), 417-437.

[4] J. M. Borwein and H. Wolkowicz, 'Characterization of optimality for the abstract convex program with finite dimensional range', J. Austral. Math. Soc. Ser. A 30 (1981), 390-411.

[5] B. D. Craven and B. Mond, 'Sufficient Fritz John optimality conditions for non-differentiable convex programming', J. A ustral. Math. Soc. Ser. B 19 (1976), 462-468.

[6] R. B. Holmes, $A$ course on optimization and best approximation (Lecture Notes in Mathematics, 247, Springer-Verlag, Berlin, Heidelberg, New York, 1972).

[7] A. D. Ioffe and V. M. Tihomirov, Theory of extremal problems (North-Holland Publishing Company, Amsterdam, 1979).

[8] P. Kanniappan and Sundaram M. A. Sastry, 'A duality theorem for non-differentiable convex programming with operatorial constraints', Bull. Austral. Math. Soc. 22 (1980), 145-152.

[9] P. Kanniappan and Sundaram M. A. Sastry, 'Duality theorems and an optimality condition for non-differentiable convex programming', J. Austral. Math. Soc. Ser. A 32 (1982).

[10] H. W. Kuhn and A. W. Tucker, Non-linear programming, Proceedings of Second Berkeley Symposium on Mathematical Statistics and Probability, pages 481-492 (University of California Press, 1951). 
[11] B. Mond, 'A class of non-differentiable mathematical programming problems', J. Math. Anal. Appl. 46 (1974), 169-174.

[12] B. Mond and M. Schechter, 'A programming problem with an $L_{p}$ norm in the objective function', J. Austral. Math. Soc. Ser. B 19 (1976), 333-342.

[13] B. Mond and S. Zlobec, 'Duality for non-differentiable convex programming, Utilitas Math. 15 (1974), 291-302.

[14] A. L. Peressini, Ordered topological vector spaces (Harper and Row, 1967).

[15] A. P. and J. W. Robertson, Topological vector spaces (Cambridge University Press, 1964).

[16] R. T. Rockafellar, Convex analysis (Princeton University Press, 1970).

[17] R. T. Rockafellar, 'Extension of Fenchel's duality theorem for a convex functions', Duke Math. J. 33 (1966), 81-89.

[18] M. Schechter, 'A subgradient duality theorem', J. Math. Anal. Appl. 61 (1977), 850-855.

[19] M. Schechter, 'More on subgradient duality', J. Math. Anal. Appl. 71 (1979), 251-262.

[20] G. A. Watson, 'A class of programming problems whose objective function contains a norm', $J$. Approximation Theorem 23 (1978), 401-411.

[21] P. Wolfe, 'A duality theorem for non-linear programming', Quart. Appl. Math. 19 (1961), 239-244.

[22] J. Zowe, 'Subdifferentiability of convex functions with values in an ordered vector spaces', Math. Scand. 34 (1974), 69-83.

School of Mathematics

Madurai Kamaraj University

Madurai-625 021

Tamil Nadu, India

Department of Mathematics

Gandhigram Rural Institute

Gandhigram-624 302

Tamil Nadu, India 